\title{
Abuso sexual em crianças e adolescentes: revisão de
} 100 anos de literatura

\section{Sexual abuse in children and adolescents: review of 100 years of literature}

\author{
Naura Liane de Oliveira Aded ${ }^{1}$ \\ BRuno Luís Galluzzi DA Silva \\ DALCIN $^{2}$
}

Talvane Marins de Moraes ${ }^{3}$

Maria Tavares Cavalcanti ${ }^{4}$

\begin{abstract}
Resumo
Maus tratos contra crianças e adolescentes, descritos por Ambroise Tardieu há cerca de um século, tornaram-se objeto de estudo nos últimos quarenta anos. $\mathrm{O}$ abuso sexual, uma das formas mais danosas, é conhecido desde a Antiguidade, porém, pouco se avançou no sentido de prevenir e amenizar suas conseqüências. Diferenças culturais, legais e de procedimentos dos profissionais envolvidos talvez expliquem a dificuldade em se estabelecer políticas públicas de prevenção e enfrentamento do problema no mundo inteiro. O presente estudo realizou uma revisão de publicações sobre abuso sexual contra menores em medicina legal e psiquiatria nos últimos cinco anos. A pesquisa, além dos artigos obtidos nas bases Medline/PubMed e Scielo, utilizou capítulos de livros e textos disponíveis on-line e em bibliotecas. Houve unanimidade entre os autores sobre os malefícios à saúde mental das vítimas e sua adaptação e inserção sociais. É patente a importância do exame médicolegal e dos procedimentos policial e judiciário para o estabelecimento da verdade sobre a ocorrência do abuso sexual. Mais estudos são necessários para conhecer essa realidade: prevalência, incidência, desdobramentos legais e consequiências futuras.
\end{abstract}

Palavras-chave: Abuso sexual na infância, agressor sexual, maus tratos na infância, perícia médico-legal, psiquiatria.

\section{Recebido: 20/09/2005 - Aceito: 16/11/2005}

1 Mestranda do Instituto de Psiquiatria da Universidade Federal do Rio de Janeiro (IPUB/UFRJ), peritolegista da Polícia Civil do Estado do Rio de Janeiro, professora auxiliar do Departamento de Patologia do Centro de Ciências Médicas da Universidade Federal Fluminense (UFF).

2 Perito-legista da Polícia Civil do Estado do Rio de Janeiro, professor assistente do Departamento de Ciências Fisiológicas da UFRJ.

3 Professor livre-docente e doutor em psiquiatria pela Escola de Medicina e Cirurgia do Rio de Janeiro da Universidade do Rio de Janeiro (Unirio), professor titular de medicina legal no curso de advocacia criminal, pós-graduação, da Universidade Candido Mendes, perito-legista aposentado da Polícia Civil do Estado do Rio de Janeiro.

4 Doutora em psiquiatria pelo IPUB/UFRJ, professora adjunta do Departamento de Psiquiatria e Medicina Legal da Faculdade de Medicina da UFRJ, professora da pós-graduação em psiquiatria e saúde mental (Propsam) do IPUB/UFRJ

Endereço para correspondência: Maria Tavares Cavalcanti. Avenida Portugal, 884, apto. 101, Urca -22291-050 - Rio de Janeiro, RJ. E-Mail: mtavares@ipub.ufrj.br 


\begin{abstract}
Child and adolescent abuse, as described by Ambroise Tardieu about a century ago, became a subject matter in the last forty years. Sexual abuse, one of the most damaging forms of maltreatment, has been known since Antiquity, but very little has been attained as to the prevention and attenuation of its consequences. Cultural differences, as well as legal questions and those regarding procedures from the involved professionals would perhaps explain the difficult to establish public polices for the world wide prevention and resolution of problem. The present study has achieved a literature review of medico-legal and psychiatric publications on the sexual abuse of minors, comprehending the last
\end{abstract}

\section{Introdução}

Os abusos sexuais têm sido descritos desde a Antiguidade. O imperador romano Tibério, segundo obra de Suetônio sobre a vida dos Césares, tinha inclinações sexuais que incluíam crianças como objeto de prazer. Há relato de que ele se retirou para a ilha de Capri com várias delas, e que as obrigava a satisfazer sua libido através da prática de diversas formas de atos sexuais (Carter-Lourensz e Johnson-Powell, 1999).

Um século separa o primeiro trabalho científico publicado sobre maus-tratos contra crianças e adolescentes e sua inclusão como assunto de interesse das áreas de pediatria e saúde pública.

A primeira monografia descrevendo a síndrome da criança espancada, Étude médico-légale sur les sevices et mauvais traitements exercés sur des enfants, foi escrita por Ambroise Tardieu, médico-legista francês, em 1860 (Roche et al., 2005). O mesmo autor, já em 1857, em Étude médico-légale sur les attentats aux moeurs, analisara 632 casos de abuso sexual de mulheres, em sua maior parte meninas, e 302 contra meninos e jovens do sexo masculino, descrevendo os sinais físicos conforme a gravidade do caso. No Dictionnaire d'hygiène et de salubrité, de 1862, Tardieu descreveu quase todas as formas de maus-tratos conforme são conhecidos hoje. O que ele infelizmente não conseguiu foi convencer seus pares de que o abuso e os maus-tratos contra crianças e adolescentes aconteciam não só no ambiente de fábricas, minas e estabelecimentos escolares, mas também no seio das famílias (Labbé, 2005).

Influenciado pelo trabalho de Tardieu, Sigmund Freud publicou um texto em 1896 no qual afirmava que a etiologia da histeria estava nos abusos sexuais da infância. O trabalho foi mal recebido nos meios acadêmicos. No ano seguinte, 1897, Freud abandonou essa teoria, explicando as memórias de abuso sexual como fantasias, conforme sua teoria do complexo de Édipo (Masson, 1984; Rocha, 2003; Labbé, 2005). five years. The search, in addition to scientific papers through the Medline/PubMed and Scielo databases, has employed textbook chapters as well as loose texts, located either in the Web or in libraries. All the sources unanimously agreed as to the noxious effects of maltreatments upon the victims' mental health, social adaptation and inclusion. The importance of the medico-legal examination and police and judicial procedures stands out in the truthful determination of sexual abuse. More studies are needed to reveal the prevalence, incidence, legal developments and future consequences of sexual abuse.

Key-words: Psychiatry, child abuse, child sexual abuse, forensic examination, sexual offender.

Segundo Ariès (1981), as crianças somente passaram a ter um papel social de relevância a partir do final do século XVII. Suas alusões ao abuso sexual freqüentemente eram consideradas "fantasiosas" ou mesmo mentirosas pelas cortes judiciais, pressupondo o desejo de prejudicar os acusados a fim de conseguir alguma vantagem (Masson, 1984).

Foram precisos mais cem anos de sofrimento para que o trabalho do médico francês fosse confirmado por um grupo de radiologistas americanos, os doutores Kempe, Silverman, Steele, Droegemueller e Silver, que, em 1962, publicaram o artigo The Battered-Child Syndrome (Krugman e Leventhal, 2005; Leventhal, 2003), que determinou nos Estados Unidos e em outros países do mesmo hemisfério a alteração de leis e a criação de políticas públicas visando o atendimento, à proteção e à prevenção do abuso e de maus-tratos contra menores.

Em todo o mundo, muito se tem escrito e estudado sobre o tema nos últimos quarenta anos, porém, pouco se avançou na prática. Talvez a magnitude do assunto ou a dificuldade que as pessoas têm em controlar o sofrimento frente a casos de maus-tratos explique $o$ fenômeno, uma vez que ocorrem fora da realidade conhecida e vivida por grande parte da sociedade, sendo encarados como "problema dos outros" (Krugman e Leventhal, 2005).

Várias são as formas de maus-tratos contra crianças e adolescentes: abusos físico, sexual, psicológico, negligência etc. Delas, não se sabe qual é a mais danosa. Questões relacionadas à reação da vítima, à situação da família nos casos de denúncia e comprovação dos abusos, bem como o desenrolar dos procedimentos policiais e judiciários, se impõem. Faz-se necessário também conhecer as repercussões na vida de crianças e adolescentes: rendimento escolar, adaptação social, alterações da saúde física e mental e a possibilidade de desenvolverem distúrbios comportamentais.

Vários transtornos psiquiátricos têm sido relacionados a eventos traumáticos sofridos na infância, com níveis de gravidade que variam com o tipo de abuso, 
sua duração e o grau de relacionamento da vítima com o agressor. Alguns estudos apontam os traumas de infância como responsáveis por cerca de $50 \%$ das psicopatologias encontradas nos adultos (Craine $e t$ al., 1988, apud Zavaschi et al., 2002). O comprometimento da saúde mental e a futura adaptação social das vítimas variarão de indivíduo para indivíduo, conforme o tipo de violência sofrida e a capacidade de reação diante de fatos geradores de estresse.

A violência, por vezes mal percebida pelas partes envolvidas, pode ser desencadeada por diversos fatores, manifestando-se de formas diferentes; daí seu grande potencial de dano.

Os tipos de abuso contra crianças mais comuns e de mais fácil detecção médico-legal são a violência física e a sexual.

O presente trabalho se propõe a revisar a literatura sobre maus-tratos na infância, focalizando o abuso sexual e sua abordagem, principalmente nas áreas de medicina legal e psiquiatria.

\section{Método}

Foi realizada busca on-line, em que as bases de dados foram a Medline-PubMed e o Scielo, com foco principal na literatura dos últimos cinco anos. Os textos foram pesquisados por área de interesse: medicina legal e psiquiatria, usando basicamente os termos de busca child abuse e child sexual abuse, sex offender e forensic examination.

Foram também consultados livros-textos e periódicos disponíveis nas bibliotecas do campus Gragoatá e Centro de Ciências Médicas da Universidade Federal Fluminense (UFF) e do Instituto de Psiquiatria da Universidade Federal do Rio de Janeiro (IPUB/UFRJ).

A escassez de trabalhos de autores brasileiros sobre o abuso infantil, no que tange à dificuldade de caracterizar sua consumação, o acompanhamento dos casos notificados às autoridades e a realidade das ocorrências policiais (no caso do presente estudo, no município do Rio de Janeiro), justifica a inclusão de trabalhos acessados em sites diversos da base consultada: www.psicopedagogia.com.artigos...; http://www. violenciasexual.org.texto...; www.unaberta.ufsc.br/...; http://www.cfch.ufrj.br... e http://kplus.cosmo.br/..., e de dados obtidos em publicação de circulação restrita, caso do Programa Delegacia Legal da Secretaria de Segurança Pública do Estado do Rio de Janeiro.

\section{Revisão da literatura}

Abusos contra crianças e adolescentes somente passaram a ser assunto de estudo e pesquisa há cerca de 45 anos, apesar de serem perpetrados desde a Antiguidade (Krugman e Leventhal, 2005; Leventhal, 2003; Roche et al., 2005; Labbé, 2005) e atingirem todas as classes socioeconômicas.
Autores têm considerado fundamental a influência da família e sua desestruturação como preditores para a criminalidade (Gover e MacKenzie, 2003; Craissati et al., 2002), o abuso de substâncias e as perturbações da saúde mental. Afirmam também que crianças expostas a um acúmulo de riscos estruturais e sociais têm maior probabilidade de manifestar problemas mentais (Arboleda-Flórez e Wade, 2001), tais como depressão na adolescência (Meyerson et al., 2002).

Os abusos físico e sexual têm sido relacionados à prática de delitos. O segundo, aos crimes da esfera sexual, enquanto o abuso físico vem sendo ligado a outros tipos de violência (Radosh, 2002; Lindsay et al., 2001).

Jovens delinqüentes mantidos em instituições penais freqüentemente provêm de cenários familiares caracterizados por abuso, negligência e outras experiências traumatizantes (Gover e MacKenzie, 2003). O abuso sexual na infância é considerado um fator de risco independente para um comportamento delinquiente (Swanston et al. 2003), com maior prevalência entre os abusadores sexuais reincidentes (Hanson e Harris, 1998, apud Aylwin et al., 2003).

$\mathrm{O}$ abuso sexual na infância é visto como fator de risco para a vitimização sexual na idade adulta, independentemente da atuação familiar (Messman-Moore e Brown, 2004), e para o desenvolvimento de psicopatologias futuras (Molnar et al., 2001).

Estudo realizado com prostitutas que atuavam em Miami, Estados Unidos ( $\mathrm{n}=325)$, demonstrou, que $50,5 \%$ das mulheres entrevistadas haviam sido vítimas de abuso sexual quando crianças. Um total de 51,7\% dessas mesmas mulheres referiu ter sofrido violência (espancamento, estupro, ameaça com armas) por parte de seus clientes no ano em que iniciaram o trabalho nas ruas (Surrat et al., 2004).

Estudos recentes têm relacionado diversas parafilias ao abuso sexual continuado na infância; quanto mais frequiente e persistente, piores os problemas psíquicos, comportamentais e de relacionamento. As seqüelas levariam as vítimas de abuso a comportamentos criminosos de transgressão dos costumes (Sharma, 2003).

A repetição dos maus-tratos em crianças pode servir de indicador de disfunção familiar. Serviços que prestam atendimentos a menores vítimas de abuso sexual devem considerar o conjunto dos fatores de risco associados à sua continuação, a fim de prevenir ocorrências futuras (Swanston et al., 2002). Os núcleos familiares de mulheres vítimas de abuso continuado na infância mostraram-se semelhantes, independentemente da existência ou não de vínculo familiar com o agressor (Gold et al., 2004).

$\mathrm{O}$ interesse social e político sobre os direitos da criança e do menor, embora crescente, pode ser considerado paradoxal nos países democráticos ocidentais. Esses direitos foram estabelecidos internacionalmente a partir de 1924, pela Convenção de Genebra sobre os direitos da criança, estendida pela Convenção Interna- 
cional das Nações Unidas de 1959 e ratificada em 1990 pelos países signatários (Morales e Schramm, 2002). A despeito de tudo isso, o abuso sexual permanece cercado por uma barreira de silêncio e se mantém perpetuado pela ignorância (Sharma e Gupta, 2004), sendo mais comum contra o sexo feminino (Queiroz, 2003; Vaz, 2003; Ramírez, 2001). A subnotificação das ocorrências envolvendo o sexo masculino não pode ser descartada (Biheler, 2002).

Esse tipo de abuso altera a vivência da sexualidade humana, sobretudo quanto aos menores, entendendo-se esta como a "integração das dimensões somáticas, emocionais, intelectuais e sociais do ser humano" (Morales e Schramm, 2002).

O fenômeno é universal, com variações decorrentes dos diferentes padrões culturais (Korbin, 2002), com igual potencial de dano, independentemente de qual forma (Chen et al., 2004) ou justificativa o abuso sexual assuma.

Estudos realizados em países da África mostram um aspecto ainda mais cruel dessa modalidade de mautrato: as crianças correm grande risco de contaminação pelo vírus HIV e desenvolvimento da própria síndrome de imunodeficiência adquirida (Aids), uma vez que existe a crença de que manter relações sexuais com crianças pode servir como meio de prevenir o contágio (Lalor, 2004a) ou "limpar" os portadores da doença. Tais fatores devem ser considerados como capazes de aumentar o risco de casos de incesto e estupro (Lalor, 2004b).

Há que se lembrar ainda a exploração da prostituição infantil, a adoção de padrões de comportamento de outras culturas, a pobreza gerando a necessidade de rápida ascensão social, o modelo patriarcal dessas sociedades e a negligência das próprias famílias e comunidades sobre o assunto. Coincidindo com o que se observa na literatura internacional, os perpetradores são, na maioria dos casos, familiares, amigos ou vizinhos, pessoas que fazem parte do universo da criança (Lalor, 2004a; Grossin et al., 2003; Morales e Schramm, 2002).

Um trabalho sobre a ocorrência de abuso sexual em Bangladesh concluiu que a maioria dos ataques ocorre na faixa entre 12 e 15 anos de idade. Resultado semelhante foi observado em estudos realizados na Índia e em países desenvolvidos, com algumas pequenas variações que podem ser atribuídas aos critérios médico-legais, à frequiência dos relatos ou mesmo aos padrões culturais (Islam e Islam, 2003).

Estudo publicado nos Estados Unidos em 1994, com base no ano de 1993 , revelou que $85 \%$ a $90 \%$ dos pacientes com problemas psiquiátricos foram vítimas de algum tipo de mau-trato na infância, com predominância do abuso sexual (Carter-Lourensz e Johnson-Powell, 1999).

Mudanças súbitas e extremas, tais como distúrbios alimentares (Gerko et al., 2005) e afetivos, comportamentos agressivos ou de autodestruição e pesadelos, podem ser observados em crianças e adolescentes em situação de abuso sexual.
Atitudes que demonstrem conhecimento sobre sexo serão mais comuns em crianças pequenas vítimas de abuso sexual que o comportamento sexual inadequado (Brilleslijper-Kater et al., 2004; O’Keefe, 2004; Biheler, 2002). A exploração sexual na infância pode levar a vítima a desenvolver uma visão sexualmente degradada de si mesma (Van Brunschot e Brannigan, 2002).

Medo, perda de interesse pelos estudos e brincadeiras, dificuldades de se ajustar, isolamento social, déficit de linguagem e aprendizagem, distúrbios de conduta, baixa auto-estima (Gerko et al., 2005; Meyerson et al., 2002), fugas de casa, uso de álcool e drogas, idéias suicidas e homicidas, tentativas repetidas de suicídio, automutilação (Ystgaard et al., 2004), agressividade e suicídio (Oates, 2004) também têm sido descritos. A dificuldade em fixar memórias relativas ao abuso pode estar presente em crianças menores, entre 3 e 10 anos de idade (Goodman et al., 2001).

Meninos que sofram abuso sexual poderão apresentar maior risco de comportamentos autodestrutivos e tentativas de suicídio, mesmo em ausência de depressão ou sentimentos de desesperança, ao contrário das meninas (Martin et al., 2004). A tristeza e a ansiedade na infância que podem levar à depressão no adulto (Conway et al., 2004; Hill et al., 2001) têm sido relacionadas ao abuso sexual.

Alterações físicas como hemorragias vaginais e/ou retais, dores ao urinar ou evacuar, infecções genitais, vômitos e dores de cabeça sem explicação médica (Junqueira e Deslandes, 2003; Vaz, 2003; Queiroz, 2003; Biheler, 2002) também devem despertar a atenção de pais, responsáveis, professores, médicos e outros profissionais de saúde.

Uma criança que tenha sido abusada sexualmente será traumatizada por toda a vida, mas a ajuda especializada somente será procurada nos casos em que, futuramente, os traumas emocionais e psicológicos venham a se agravar (Sharma e Gupta, 2004). As seqüelas poderão variar quando relacionadas ao tipo de abuso: número de agressores, duração, relação com agressor, resistência, emprego de força, idade em que se iniciou, participação e frequiência do abuso (Steel et al., 2004). O abuso sexual aumenta o risco de suas vítimas desenvolverem transtorno de estresse pós-traumático (PTSD) e apresentarem comportamentos autodestrutivos. Dificuldade nos relacionamentos sexuais também é descrita (Peleikis et al., 2004; Queiroz, 2003). Transtornos dissociativos (Grande et al., 2003; Zavaschi et al., 2002; Kisiel e Lions, 2001), fobias, ideação paranóide e transtorno obsessivo-compulsivo têm sido referidos ao abuso sexual em crianças, adolescentes e adultos, podendo variar conforme $\mathrm{o}$ agressor e a idade em que ocorra o abuso (Haj-Yahia e Tamish, 2001; Macfie et al., 2001). Transtorno de conversão tem sido relacionado a pacientes com história de abuso sexual de longa duração e relações incestuosas (Ystgaard $e t$ al., 2004; Roelofs et al., 2002). 
$\mathrm{O}$ abuso sexual tem consequiências psíquicas que vão além daquelas causadas pelo fato em si. Elas se referem, direta ou indiretamente, aos efeitos do processo legal e seus desdobramentos (Ghetti et al., 2002).

Estudo feito na Costa Rica demonstrou que os casos de abuso sexual ocorrem predominantemente na faixa etária dos 6 aos 14 anos para ambos os sexos (Ramírez, 2001). Crianças mais novas (menores de 6 anos) e adolescentes (principalmente do sexo feminino) na faixa dos 13 aos 17 anos têm sido registrados como vítimas de abuso sexual (Barros, 2004; Suarez Sola e Gonzalez Delgado, 2003b).

Repercussões duradouras na saúde mental, dificuldade no relacionamento com os filhos e de adaptação a problemas ao longo do tempo foram relacionados ao abuso ocorrido antes dos 13 anos (Roberts et al., 2004).

Estimativas de prevalência e incidência da violência sexual contra crianças e adolescentes e a relação destes fatores com o seu cotidiano são fundamentais para o desenvolvimento de políticas de prevenção e abordagem do problema.

Os dados disponíveis sobre o abuso sexual no País não enfocam o testemunho ou a convivência com as vítimas. São fundamentados quase exclusivamente em relatos de entidades governamentais que não alcançam a real dimensão do problema. Gomes (1998) destacou a dificuldade encontrada para acompanhar casos selecionados a partir de um recorte nos dados gerais de pesquisa realizada pelo Centro Latino-Americano de Estudos sobre Violência e Saúde da Fundação Oswaldo Cruz, de 1994, em visitas às delegacias policiais, a fim de verificar, após cinco anos, o encaminhamento dado aos registros das denúncias. Entre os problemas relatados, destacaram-se o arquivamento das fichas em local de difícil acesso e o fato de algumas delegacias não facilitarem a tarefa. Somente 106 casos de violência doméstica na faixa de zero a 5 anos foram localizados: $80 \%$ de casos relacionados à morbidade e $20 \%$ de casos fatais. Desse total, apenas 24 foram transformados em inquérito; desses, somente 1, por se tratar de homicídio, tornou-se processo, sendo o pretenso agressor absolvido. Um total de $31 \%$ dessas vítimas foi encaminhado a exame pericial para fundamentação das denúncias. O autor do estudo frisou o sentido de maus-tratos e negligência contidos nas agressões físicas.

Fatores como a não-notificação das ocorrências às autoridades policiais, por medo de represálias ou do estigma social, dificultam o conhecimento do desfecho desses casos (Polanczyk et al., 2003).

O tempo entre o fato e a comunicação às autoridades pode variar, sendo mais curto nos casos em que o agressor é desconhecido ou não tem relação de parentesco com a vítima. Nos casos em que o agressor é o próprio pai, ou familiar, a denúncia pode demorar (Sharma e Gupta, 2004), provavelmente até que a criança perceba que tais práticas são incorretas ou injustas, ou que possa se defender. Em amostra pesquisada na cidade de Santa Cruz de Tenerife (Ilhas Canárias), 67\% das denúncias foram feitas pela própria ofendida, e $88 \%$ dos perpetradores eram conhecidos (Suarez Sola e Gonzalez Delgado, 2003b).

Podem ainda ser considerados motivos para a manutenção desse tipo de equívoco: a falta de preparo profissional para a identificação dos casos de maus-tratos, desconhecimento das leis, descrédito nas possíveis ações do Estado para resolver o problema, banalização dos efeitos sobre as vítimas pela violência sofrida, além do descrédito no relato da vítima (Paiva, 2000). Adolescentes abusados sexualmente teriam dificuldade em se abrir com membros da família por receio de não serem levados a sério, vergonha ou medo dos problemas que tal revelação pudesse causar no meio familiar (Koshima, 1999). O desconhecimento da existência e o funcionamento de serviços de assistência, a vontade de manter o problema em segredo, a não percepção do abuso e a desconfiança inspirada por adultos e/ou profissionais dificulta a procura por atendimento especializado. Outro fator que pode ser considerado é a limitação do amparo que eles podem oferecer a esses menores (Crisma et al., 2004).

Profissionais de saúde mental têm, por sua vez, dificuldade em substanciar ou não os casos suspeitos de abuso sexual quando solicitados a auxiliar nas decisões judiciais. Eles se valem para isso de dados obtidos em exames médico-legais, testes psicológicos, revisões e sumários de pesquisas relevantes na área das ciências sociais. Análise sobre o assunto concluiu que pelo menos $24 \%$ dessas decisões tanto podem resultar em avaliações falso-positivas como falso-negativas (Herman, 2005).

Além do baixo número de notificações - estima-se que somente $10 \%$ dos casos sejam notificados (Barros, 2004; Santos, 1998) - o que preocupa é o altíssimo número de casos de maus-tratos que são diagnosticados e/ou qualificados como outro tipo de crime. O depoimento de um atendente de uma Delegacia Especializada de Atendimento à Mulher, no estado do Rio de Janeiro, ilustra a dificuldade para conhecer a realidade dos maus-tratos em geral: o funcionário acha que o exame de corpo de delito, no caso, para comprovação de violência sexual, "não vai conseguir provar nada que auxilie no andamento das investigações", e ainda acrescentou: "É bobeira mandar a vítima pro [sic] IML à toa, é melhor registrar o crime como tentativa ou ameaça" (Paiva, 2000).

As falhas nos registros podem ser atribuídas também a fatores médicos: desconhecimento do profissional, associado ou não a razões culturais: o médico rechaçaria a possibilidade de o dano ter sido produzido por pessoas próximas e/ou responsáveis pela criança. Outros motivos que levariam o médico a não se pronunciar: "razões éticas; contato estreito com a comunidade; medo de revanchismo contra bens, família ou contra si; medo de aparecer na imprensa; temor de transtornos legais e acusação de falsa denúncia; temor de comparecimento ao tribunal com perda de tempo profissional" (Santos, 1998). 
É importante destacar que adolescentes vítimas e testemunhas de atos de violência sexual encontram-se com frequiência mais expostos à violência comunitária em geral, comparados com os que não foram expostos à violência sexual. A exposição a outros tipos de violência seria uma consequiência do comportamento desorganizado possivelmente associado ao evento traumático (Polanczyk et al., 2003). Experimentar ou testemunhar atos violentos no ambiente familiar, durante a infância tem sido associado à delinquiência e à violência juvenis, tanto quanto às várias formas de agressões contra mulheres (White e Smith, 2001).

Cabe ressaltar que algumas crianças, apesar do sofrimento causado pelos maus-tratos, passam por essa experiência sem apresentar o quadro de seqüelas descrito pela literatura especializada, conseguindo encontrar "caminhos de (re)construção de suas próprias vidas" (Junqueira e Deslandes, 2003).

O exame médico-legal é de suma importância para o levantamento das lesões físicas, seqüelas emocionais e mesmo a avaliação da credibilidade das vítimas (Ramírez, 2001; Lipian et al., 2004; Calçada et al., 2002).

A experiência médico-legal tem demonstrado ser difícil caracterizar diferentes formas de maus-tratos contra crianças e adolescentes, mormente o abuso sexual (Aded e Oliveira, 1997; Aded, 2005), tomando por base somente o exame pericial (exame de corpo de delito). A pobreza das informações contidas no documento policial de encaminhamento ao Instituto Médico-Legal e na anamnese do perito-legista, observada ao longo do levantamento de casos da presente pesquisa, merece destaque.

Outro fator que aumenta a dificuldade do diagnóstico, nos casos de suspeita de abuso sexual, é a ausência de lesões físicas filiáveis à alegação, muitas vezes por causa do tempo decorrido entre o fato e o exame pericial (Suarez-Sola e Gonzalez Delgado, 2003b; Aded e Oliveira, 1997; Aded, 2005). Exames normais não excluem o abuso. A maior parte de abusos sexuais confirmados em crianças impúberes não evidenciam lesões, ou apenas revelam achados inespecíficos (Pillai, 2005). A taxa de exames normais oscila entre $16 \%$ e $90 \%$, havendo estudos que relatam de 70\% a 90\% (O'Keefe, 2004), mesmo naqueles em que o agressor confessa a prática.

Encontro de sêmen, esperma, exames laboratoriais positivos para doenças sexualmente transmissíveis e para o vírus HIV podem ser uma evidência do abuso, mesmo que não haja um histórico a esse respeito, desde que excluídas outras formas de contágio (Suarez Sola e González Delgado, 2003a; Aded e Oliveira, 1997; Aded, 2005). Há que se considerar ainda o encontro de lesões que tanto podem ser devidas ao abuso sexual quanto a acidentes ou patologias, suscitando dúvidas e requerendo pesquisa atenta a fim de definir sua etiologia (Makoroff et al., 2002; Jesus et al., 2001; Dmitrieva, 2003; Aded e Oliveira, 1997; Aded, 2005).

Pediatras, por vezes desavisados da gravidade do problema e de sua repercussão sobre as vidas da criança e família, não raro concluem por diagnósticos equivocados de maus-tratos, causando sérios problemas para os envolvidos. A demora em reconhecer sinais de abuso sexual e/ou físico, por outro lado, poderá causar graves consequiências (Özkara et al., 2004).

A interpretação de achados anormais na genitália de crianças atendidas por pediatras nas emergências dos hospitais freqüentemente se mostra em desacordo com a interpretação desses mesmos sinais por peritoslegistas, mais treinados na observação de tais alterações (O’Keefe, 2004; Makoroff et al., 2002). Há que se considerar que os peritos estejam menos expostos ao conflito de interesses presente nos casos suspeitos de abuso sexual, o mesmo não acontecendo com os pediatras, que, por sua atuação terapêutica, se vêem envolvidos com crianças e pais (Pillai, 2005). Não raro são encaminhados para exame médico-legal, sempre precedido pela ida à delegacia policial e pelo registro da ocorrência (com as repercussões esperadas para o menor e seu responsável), casos clínicos que poderiam ser elucidados por exame mais atento e menos receoso por parte do pediatra.

Os desencontros entre os diagnósticos feitos por pediatras e peritos-legistas talvez possam ser explicados pelo resultado de um levantamento sobre a produção científica na área de pediatria nos anos 1990, realizado em 2002, que encontrou apenas 14 artigos sobre maustratos contra crianças em três das principais revistas de pediatria do País. Os autores concluíram pela necessidade de mais dados ligados à realidade brasileira, uma vez que os indicadores utilizados nas discussões sobre o assunto são construídos em ambientes socioculturais diversos do nosso. Outra conclusão foi a constatação de que o tema foi pouco abordado pela especialidade, demonstrando que a produção não acompanhou a demanda na área de promoção de saúde infantil, que é compreender a violência cometida contra a criança, para que possam ser sugeridas políticas de atenção e enfrentamento do problema (Gomes et al., 2002).

A veracidade ou a falsidade do abuso sexual deverá ser investigada. Interpretações ou memórias equivocadas por parte da criança e submissão ao adulto que levem o menor a mentir deliberadamente sobre o suposto abuso sexual e a formular falsas denúncias não são raras (Lipian et al., 2004), cabendo aos profissionais envolvidos manter o distanciamento necessário à apuração dos fatos - daí a necessidade de um trabalho multidisciplinar (Pillai, 2005; Calçada et al., 2002).

\section{Discussão}

A preocupação que as consequiências do abuso sexual contra crianças e adolescentes despertam é demonstrada pela grande quantidade de artigos publicados em todo o mundo.

Muito se tem escrito sobre as seqüelas decorrentes das diversas formas de abuso, mas os esforços para 
proteger essas crianças não se têm mostrado suficientes (Krugman e Leventhal, 2005).

Há carência de ações efetivas na condução e na prevenção do problema, uma vez que as pesquisas sobre sua ocorrência mostram-se semelhantes, tanto no Brasil e outros países em desenvolvimento, como nos considerados desenvolvidos (Islam e Islam, 2003).

É difícil conhecer a real extensão desse tipo de agressão, tendo em vista a subnotificação dos casos de abuso sexual (Biheler, 2002; Lalor, 2004 A; Polanczyk et al., 2003; Suarez-Sola e Gonzalez Delgado, 2003b; Barros, 2004; Koshima, 1999; Santos, 1998), as diferenças culturais (Korbin, 2002; Lalor, 2004a; Islam e Islam, 2003) e de legislação, bem como a conduta dos profissionais (peritos-legistas, médicos, assistentes sociais, autoridades policiais e judiciárias) envolvidos, que não raro resulta em diagnósticos e análises equivocados (Paiva, 2000; Herman, 2005; Santos, 1998).

A escassez e a irregularidade dos serviços de assistência, o amparo limitado oferecido às vítimas (Crisma et al., 2004) e a dificuldade em acompanhar os casos registrados como abuso (Gomes, 1998; Polanczyk et al., 2003) concorrem para que o assunto permaneça pouco conhecido.

Em que pese a unanimidade dos autores sobre as repercussões que o abuso sexual e outros tipos de violência determinem na saúde mental (Zavaschi et al., 2002; Arboleda-Flórez e Wade, 2001; Meyerson et al., 2002; Molnar et al., 2001; Conway et al., 2004; Hill et al., 2001; Sharma e Gupta, 2004; Steel et al., 2004; Peleikis et al., 2004; Queiroz, 2003; Grande et al., 2003; Kisiel e Lyons, 2001; Haj-Yahia e Tamish, 2001; Macfie et al., 2001; Ystgaard et al., 2004; Roelofs et al., 2002; Roberts et al., 2004), além da predisposição para um comportamento delinquiente (Gover e MacKenzie, 2003; Craissati et al., 2002; Radosh, 2002; Lindsay et al., 2001; Swanston et al., 2003; Sharma, 2003; White e Smith, 2004), seja em idade adulta (Aylwin et al., 2003) ou na juventude, não há consenso a respeito da idéia de que a criança vítima de violência será um adulto violento, capaz de práticas abusivas (Craissati et al., 2002). O abuso sexual na infância é considerado também como fator de risco para a vitimização na idade adulta (Messman-Moore e Brown, 2004; Surrat et al., 2004; Polanczyk et al., 2003).

A presente revisão levantou 18 trabalhos de autores brasileiros, somente 7 deles publicados em revistas indexadas.

\section{Conclusão}

Há necessidade de mais pesquisa no País sobre o abuso sexual contra menores: prevalência, incidência, desdobramentos legais e consequiências para a vida futura das vítimas. Esses estudos devem ser feitos de acordo com os parâmetros da realidade socioeconômico-cultural brasileira.

Pediatras e peritos-legistas devem ser alertados para o problema dos abusos na infância, tornando-se aptos a atuarem de maneira adequada e entrosada; aqueles, fornecendo as informações capazes de auxiliar a investigação médico-legal. Devem ser criadas e mantidas equipes multidisciplinares, capazes de lidar com os diversos aspectos do problema. Há necessidade de mudanças nas estruturas policial e judiciária, com o objetivo de possibilitar o seguimento dos casos a partir do registro policial e do exame médico-legal.

\section{Referências bibliográficas}

Aded, N.L.O. - Síndrome da Criança Espancada, In: Hércules, H. (ed.) Medicina Legal Texto e Atlas. Atheneu, Rio de Janeiro, pp. 641-652, 2005.

Aded, N.L.O.; Oliveira, S.F. - Síndrome da Criança Espancada, In: Hércules, H. (atual.) Medicina Legal Hélio Gomes. Ed. Freitas Bastos, Rio de Janeiro, pp. 725-742, 1997.

Arboleda-Flórez, J.; Wade, T.J. - Chilhood and adult vitimization as risk factor for major depression. International Journal of Law and Psychiatry 24: 357-370, 2001.

Ariès, P. - História Social da Criança e da Família. LTC, Rio de Janeiro, pp. 9-27, 1981.

Aylwin, A.S.; Studer, L.H.; Reddon, J.R.; Clelland, S.R. - Abuse prevalence and victim gender among adult and adolescent child molesters. International Journal of Law and Psychiatry 26: 179-190, 2003.
Barros, W.S. - Crimes contra os costumes - Estupro. Programa Delegacia Legal, Secretaria de Segurança Pública, Governo do Estado do Rio de Janeiro, Rio de Janeiro; pp. 14-19, 2004.

Biheler, S. - As pessoas não estão preparadas para lidar com o abuso sexual, porque a sexualidade assusta. Universidade Federal de Santa Catarina, 2002. Disponível em www.unaberta.ufsc.br/entrevista_ abuso.html. Acesso em 15 de janeiro de 2003.

Brilleslijper-Katwer, S.; Friedrich, W.N.; Corwin, D.L. - Sexual knowledge and emotional reaction as indicators of sexual abuse in young children: theory and research challenges. Child Abuse \& Neglect 28: 1007-1017, 2004.

Calçada, A.; Cavaggioni, A.; Néri, L. - 0 uso do abuso sexual - o outro lado da história. Psicopedagogia On Line, 2002. 
Disponível em http://www.psicopedagogia.com.br/artigos/print.asp?entrID=333. Acesso em 10/03/2003.

Carter-Lourensz, J.H.; Johnson-Powell, G. - Abuso Físico, Abuso Sexual e Negligência da Criança, In: Kaplan, H.I.; Saddock, B.J. Tratado de Psiquiatria, v. 3. Artmed, Porto Alegre, pp. 2660-2676, 1999.

Chen, J.0.; Dunne, M.P.; Han, P. - Child abuse in China: a study of adolescents in four provinces. Child Abuse \& Neglect 28 (11): 1171-1186, 2004.

Conway, M.; Mendelson, M.; Giannopoulos, C.; Csank, P.A.R.; Holm, S.L. - Childhood and adult sexual abuse, rumination on sadness, and dysphoria. Child Abuse \& Neglect 28 : 393- 410, 2004.

Craissati, J.; McClurg, G.; Browne, K. - The parenteral bonding experiences of sex offenders: a comparison between child molesters and rapists. Child Abuse \& Neglect 26 (9): 909-921, 2002.

Crisma, M.; Bascelli, E.; Paci, D.; Romito, P. - Adolescents who experienced sexual abuse: fears, needs and impediments to disclosure. Child Abuse \& Neglect 28: 1035-1048, 2004.

Dmitrieva, 0.A. - Consequences for the teenagers after anogenital sexual contacts. Legal Medicine 5 (S1): S386-S389, 2003.

Gerko, K.; Hughes, M.L.; Hamil, M.; Waller, G. - Reported childhood sexual abuse and eating-disordered cognitions and behavior. Child Abuse \& Neglect 29 (4): 375-382, 2005.

Ghetti, S.; Alexander, K.W.; Goodman, G.S. - Legal involvement in child sexual abuse cases. Consequences and interventions. International Journal of Law and Psychiatry 25: 235-251, 2002.

Gomes, R. - Da denúncia à impunidade: um estudo sobre a morbi-mortalidade de crianças vítimas de violência. Cadernos de Saúde Pública 14 (2): 301-311, 1998.

Gomes, R.; Deslandes, S.F.; Veiga, M.M.; Bhering, C.; Santos, J.F.C. - Por que as crianças são maltratadas? Explicações para a prática de maus-tratos infantis na literatura. Cadernos de Saúde Pública 18 (3): 707-714, 2002.

Gold, S.N.; Hyman, S.M.; Andrés-Hyman, R.C. - Family of origin environments in two clinical samples of survivors of intra-familial, extra-familial, and both types of sexual abuse. Child Abuse \& Neglect 28: 1199-1212, 2004.

Goodman, G.S.; Bottoms, B.L.; Rudy, L.; Davis, S.L.; SchwartzKenney, B.M. - Effects of past abuse experience on children's eyewitness memory. Law and Human Behavior 25 (3): 269-298, 2001.

Gover, A.R.; Mackenzie, D.L. - Child maltreatment and adjustment to juvenile correctional institutions. Criminal, Justice and Behavior 30 (3): 374-396, 2003.

Grande, C.Z.; Alvarado, J.G.A.; Sánchez, F.M.; Hernández, A.N. - Trastorno de estrés postraumático en adolescentes agredidos. Medicina Legal de Costa Rica 20 (2).

Grossin, C.; Sibille, l.; Lorin De La Grandmaison, G.; Banasr, A.; Brion, F.; Durigon, M. - Analysis of 418 cases of sexual assault. Forensic Science International 131 (2-3): 125-130, 2003.
Haj-Yahia, M.M.; Tamish, S. - The rates of child sexual abuse and its psychological consequences as revealed by a study among Palestinian university students. Child Abuse \& Neglect 25 (10): 1303-1327, 2001.

Herman, S. - Improving decision making in forensic child abuse evaluations. Law and Human Behavior 29 (1): 87-120, 2005.

Hill, J.; Pickles, A.; Burnside, E.; Byatt, M.; Rollinson L.; Davis R.; Harvey K. - Child sexual abuse, poor parental care and depression: evidence for different mechanisms. British Journal of Psychiatry 179: 104-109, 2001.

Islam, M. N.; Islam, M. N. - Retrospective study of alleged rape victims attended at Forensic Medicine Department of Dhaka Medical College, Bangladesh. Legal Medicine 5: S351-S353, 2003.

Jesus, L.E.; Cirne Neto, O.L.L.; Nascimento, L.M.M. et al. - Anogenital warts in children: sexual abuse or unintentional contamination? Cadernos de Saúde Pública 17 (6): 1383-1391, 2001.

Junqueira, M.F.P.S.; Deslandes, S.F. - Resiliência e maus-tratos à criança. Cadernos de Saúde Pública 19 (1): 227-235, 2003.

Kisiel, C.L.; Lyons, J.S. - Dissociation as a mediator of psychopathology among sexually abused children and adolescents. American Journal of Psychiatry 158 (7): 1034-1039, 2001.

Koshima, K. - Palavra de Criança. CEDECA - Bahia, 1999. Disponível em http://www.violenciasexual.org.br/textos/PDF/palavra_criança_karin_koshima.pdf. Acesso em novembro de 2002.

Korbin, J.E. - Culture and child maltreatment: cultural competence and beyond. Child Abuse \& Neglect 26: 637-644, 2002.

Krugman, R. D.; Leventhal, J. M. - Confronting child abuse and neglect and overcoming gaze aversion: the unmet challenge of centuries of medical practice. Child Abuse \& Neglect 29 (4): 307-309, 2005.

Labbé, J. - Ambroise Tardieu: The man and his work on child maltreatment a century before Kempe. Child Abuse \& Neglect 29 (4): 311-324, 2005.

Lalor, K. - Child abuse in sub-Saharan Africa: a literature review. Child Abuse \& Neglect 28: 439-460, 2004 A.

Lalor, K. - Child abuse in Tanzania and Kenya. Child Abuse \& Neglect 28: 833-844, 2004 B.

Leventhal, J.M. - The field of child maltreatment enters its fifth decade. Child Abuse \& Neglect 27: 1-4, 2003.

Lindsay, W.R.; Law, J.; Quinn, K.; Smart, N.; Smith, A.H.W. - A comparison of physical and sexual abuse: histories of sexual and non-sexual abuse with intellectual disability. Child Abuse \& Neglect 25 (7): 989-995, 2001.

Lipian, M.S.; Mills, M.J.; Brantmman, A. - Assessing the verity of children's allegations of abuse: A psychiatric overview. International Journal of Law and Psychiatry 27: 249-263, 2004.

MacFie, J.; Cicchetti, D.; Toth, S.L. - Dissociation in maltreated preschool-aged children. Child Abuse \& Neglect 25 (9): 1253-1267, 2001. 
Makaroff, K.L.; Brauley, J.L.; Brandner, A.M.; Myers, P.A.; Shapiro, R.A. - Genital examinations for alleged sexual abuse of prepubertal girls: findings by pediatric emergency medicine physicians compared with child abuse trained physicians. Child Abuse \& Neglect 26 (12): 12351242, 2002.

Martin, G.; Bergen, H.A.; Richardson, A.S.; Roeger, L.; Allison, $S$. - Sexual abuse and suicidality: gender differences in a large community sample of adolescents. Child Abuse \& Neglect 28: 491-503, 2004.

Masson, J.M. - Atentado à verdade. A supressão da teoria da sedução por Freud. José Olympio Editora, Rio de Janeiro, 1984. 290 p.

Messman-Moore, T.; Brown, A.L. - Child maltreatment and perceived family environment as risk factors for adult rape: is child abuse the most salient experience? Child Abuse \& Neglect 28: 1019-1034, 2004.

Meyerson, L.A.; Long, P.; Miranda Jr., R.; Marx, B.P. - The influence of childhood sexual abuse, physical abuse, family environment, and gender on the psychological adjustment of adolescents. Child Abuse \& Neglect 26: 387-405, 2002.

Molnar, B.E.; Buka, S.L.; Kessler, R.C. - Child sexual abuse and subsequent psychopathology: results from the National Comorbidity Survey. American Journal of Public Health 91 (5): 753-760, 2001.

Morales, A.E.; Schramm, F.R. - A moralidade do abuso sexual intrafamiliar em menores. Ciência \& Saúde Coletiva 7 (2): 265-273, 2002.

Oates, R.K. - Sexual abuse and suicidal behavior. Child Abuse \& Neglect 28: 487-489, 2004.

0 'Keefe, M. - A case of suspected child sexual abuse. Journal of Clinical Forensic Medicine 11: 316-320, 2004.

Özkara, E.; Karatosun, V.; Gunal, I.; Oral, R. - Trans-metatarsal amputation as a complication of child sexual abuse. Journal of Clinical Forensic Medicine 11 (3): 129-132, 2004.

Paiva, R.M. - A dimensão de gênero na violência doméstica contra a infância, 2000. Disponível em http://www.cfch. ufrj.br/jor_p4/Relacge2/dimegene.html. Acesso em 18/12/2000.

Peleikis, D.E.; Mykletun, A.; Dahl, A.A. - The relative influence of childhood sexual abuse and other family background risk factors on adult adversities in female outpatients treated for anxiety disorders and depression. Child Abuse \& Neglect 28: 61-76, 2004.

Pillai, M. - Forensic examination of suspected child victims of sexual abuse in the UK: a personal view. Journal of Clinical Forensic Medicine 12: 57-63, 2005.

Polanczyk, G.V.; Zavaschi, M.L; Benetti, S.; Zenker, R.; Gammerman, P. W. - Violência sexual e sua prevalência em adolescentes de Porto Alegre, Brasil. Revista de Saúde Pública 37 (1): 8-14, 2003.

Queiroz, K. - Abuso Sexual. Conversando com esta realidade. CEDECA-BA, 2003. Disponível em http://www.violenciasexual.org.br/texto/PDF/abuso_sexual_katia_keiroz.pdf. Acesso em 10/06/2003.
Radosh, P.F. - Reflections on women's crime and mothers in prison: a peacemaking approach. Crime \& Delinquency 48 (2): 300-315, 2002.

Ramírez, S.M.C. - Delitos sexuales valorados en el Consejo Médico Forense y su seguimiento en el proceso judicial. Medicina Legal de Costa Rica 18 (2), setembro de 2001.

Roberts, R.; O'Connor, T.; Dunn, J.; Golding, J.; The ALSPAC study Team - The effects of child sexual abuse in later family life; mental health, parenting and adjustment of offspring. Child Abuse \& Neglect 28: 525-545, 2004.

Rocha, P.P. - Os abusos na criança, In: A Saga do Autismo. Psicopedagogia On-Line. Disponível em http://216.239.57.100/ custom?q=cache:j0jinpYCJvcC:www.psicopedagogia. com.artigos.... Acesso em 11/03/2003.

Roche, A.J.; Fortín, G.; Labbé, J.; Brown, J.; Chadwick, D. - The work of Ambroise Tardieu: The first definitive description of child abuse. Child Abuse \& Neglect 29 (4): 325-334, 2005.

Roelofs, K.; Keijers, G.P.J.; Hoogduin, K.A.L.; Näring, G.W.B.; Moene, F.C. - Childhood abuse in patients with conversion disorder. American Journal of Psychiatry 159: 1908-1913, 2002.

Santos, H.O. - Síndrome da Criança Abusada e Negligenciada, In: Medicina Legal para Não Legistas. Copola, São Paulo, 1998.

Sharma, B.R. - Disorders of sexual preference and medicolegal issues thereof. American Journal of Forensic Medicine and Pathology 24 (3): 277-282, 2003.

Sharma, B.R.; Gupta, M. - Child abuse in Chandigarh, India, and its implications. Journal of Clinical Forensic Medicine 11 (5): 248-256, 2004.

Steel, J.; Sanna, L.; Hammond, B.; Whipple, J.; Cross, H. - Psychological sequelae of childhood sexual abuse: abuse-related characteristics, coping strategies, and attributional style. Child Abuse \& Neglect 28: 785-801, 2004.

Suarez-Sola, M.L.; Gonzalez Delgado, F.J. - Importancia de la exploración médico forense en las agresiones sexuales a menores. Cuadernos de Medicina Forense 31: 37-45, $2003 \mathrm{~A}$.

Suarez-Sola, M.L.; Gonzalez Delgado, F.J. - Statistics and significance of sexual violence in minors. Cuadernos de Medicina Forense 32: 49-62, 2003 B.

Surrat, H.L.; Inciardi, J.A.; Kurtz, S.P.; Kiley, M.C. - Sex work and drug use in a subculture of violence. Crime \& Delinquency 50 (1): 43-59, 2004.

Swanston, H.Y.; Parkinson, P.N.; Oates, R.K.; O'Toole, B.L.; Plunkett, A.M.; Shrimpton, S. - Further abuse of sexually abused children. Child Abuse \& Neglect 26 (2): 115-127, 2002.

Swanston, H.Y.; Parkinson, P.N.; O'Toole, B.L.; Plunkett, A.M.; Shrimpton, S; Oates, R.K. - Juvenile crime, aggression and delinquency after sexual abuse. The British Journal of Criminology 43: 729-749, 2003.

Van Brunschot, E.G.; Brannigan, A. - Childhood maltreatment and subsequent conduct disorders. The case of female 
street prostitution. International Journal of Law and Psychiatry 25: 219-234, 2002.

Vaz, M. - Abuso Sexual. CEDECA-BA, 2003. Disponível em http://www.violenciasexual.org.br/texto/PDF/abuso_sexual_marlene_vaz.pdf Acesso em 10/06/2003.

White, J.W.; Smith, P.H. - Sexual assault perpetration and reperpetration: from adolescence to young adulthood. Criminal, Justice and Behavior 31 (2): 182-202, 2004.
Ystgaard, M.; Hestetun, I.; Loeb, M.; Mehlum, L. - Is there a specific relationship between childhood sexual and physical abuse and repeated suicidal behavior? Child Abuse \& Neglect 28 (8): 863-875, 2004.

Zavaschi, M.L.S.; Satler, F.; Poester, D.; Vargas, C. F.; Piazenski, R.; Rohde, L.A.P. et al. - Associação entre trauma por perda na infância e depressão na vida adulta. Revista Brasileira de Psiquiatria 24 (4): 189-195, 2002. 